\title{
MYC gene amplification is often acquired in lethal distant breast cancer metastases of unamplified primary tumors
}

\author{
Aatur D Singhi ${ }^{1}$, Ashley Cimino-Mathews ${ }^{1}$, Robert B Jenkins ${ }^{2}$, Fusheng Lan $^{2}$, \\ Stephanie R Fink ${ }^{2}$, Hind Nassar ${ }^{1}$, Russell Vang ${ }^{1}$, John H Fetting, ${ }^{3}$, Jessica Hicks ${ }^{1}$, \\ Saraswati Sukumar ${ }^{3}$, Angelo M De Marzo ${ }^{1,3}$ and Pedram Argani ${ }^{1,3}$ \\ ${ }^{1}$ Department of Pathology, Johns Hopkins Medical Institutions, Baltimore, MD, USA; ${ }^{2}$ Department of \\ Laboratory Medicine and Pathology and Laboratory Genetics, Mayo Clinic, Rochester, MN, USA and \\ ${ }^{3}$ Department of Oncology, Johns Hopkins Medical Institutions, Baltimore, MD, USA
}

\begin{abstract}
In breast cancer, amplification of $M Y C$ is consistently observed in aggressive forms of disease and correlates with poor prognosis and distant metastases. However, to date, a systematic analysis of $M Y C$ amplification in metastatic breast cancers has not been reported. Specifically, whether the MYC amplification status may change in metastases in comparison to the corresponding primary breast tumor, and potential variability among different metastases within the same patient have also not been assessed. We generated single patient tissue microarrays consisting of both primary breast carcinomas and multiple matched systemic metastases from 15 patients through our previously described rapid autopsy program. In total, the 15 tissue microarrays contained 145 primary tumor spots and 778 spots derived from 180 different metastases. In addition, two separate tissue microarrays were constructed composed of 10 matched primary breast cancers and corresponding solitary metastases sampled not at autopsy but rather in routine surgical resections. These two tissue microarrays totaled 50 primary tumor spots and 86 metastatic tumor spots. For each case, hormone receptor status, HER2/neu, EGFR and CK5/6 expression were assessed, and the cases were characterized as luminal, basal-like or HER2 based on published criteria. Both fluorescence in situ hybridization and immunohistochemistry for $M Y C$ was performed on all cases. Of the 25 cases, 24 were evaluable. While 4 of 24 primary tumors (16\%) demonstrated MYC amplification, an additional 6 (25\% of total evaluable cases) acquired MYC amplification in their systemic metastases. Of note, there was remarkably little heterogeneity in MYC copy number among different metastases from the same patient. MYC immunoreactivity was increased in metastases relative to matched primaries in the surgical cohort, although there was no perfect correlation with MYC amplification. In conclusion, amplification of $M Y C$ is a frequent event in breast cancer, but occurs more frequently as a diffuse, acquired event in metastatic disease than in the corresponding primary. These observations underscore the importance of $M Y C$ in breast cancer progression/metastasis, as well as its relevance as a potential therapeutic target in otherwise incurable metastatic disease.
\end{abstract}

Modern Pathology (2012) 25, 378-387; doi:10.1038/modpathol.2011.171; published online 4 November 2011

Keywords: breast cancer; fluorescent in situ hybridization; MYC

Breast cancer is well known to be highly variable at both the clinical and genetic levels. Many cases progress rapidly with short survival, whereas others grow indolently with relatively good outcome after

Correspondence: Dr P Argani, MD, The Johns Hopkins Hospital, 401 N. Broadway, Weinberg 2242, Baltimore, MD 21231-2410, USA.

E-mail: pargani@jhmi.edu

Received 22 July 2011; revised 31 August 2011; accepted 31 August 2011; published online 4 November 2011 treatment. Not surprisingly, at the molecular level, breast cancer is also characterized by a diverse number of genetic abnormalities including unbalanced chromosomal rearrangements, gene amplifications and deletions. Some of these genetic alterations have been shown to be associated with tumors of distinct histological types and/or grades (such as loss of $16 \mathrm{q}$ in low-grade ductal and lobular carcinomas), whereas others have proven to be of prognostic and predictive value (HER2/neu amplification). Another common feature of breast 
cancers are structural and numerical alterations in chromosome $8 .^{1,2}$ The changes typically consist of gains on the $q$-arm, either the entire $8 \mathrm{q}$ or its telomeric region. ${ }^{3}$ These chromosomal alterations have commonly been interpreted to reflect amplification of the $M Y C$ gene, located at $8 q 24.1 .^{4}$

The proto-oncogene $M Y C$ encodes a nuclear phosphoprotein transcription factor that has an integral role in a variety of cellular processes, such as cell cycle progression, proliferation, metabolism, adhesion, differentiation and apoptosis. ${ }^{5}$ In cell culture, the activation of $M Y C$ leads to either entry into and progression through the cell cycle, or an increased rate of apoptosis, depending on the cell type and/or context. ${ }^{6}$ Further, overexpression of $M Y C$ alone confers resistance to antiestrogen treatment. $^{7}$ The breast cancer suppressor gene, BRCA1, has been found to bind to $M Y C$ and inhibit its transcriptional and transforming activity. ${ }^{8}$ Experiments in transgenic mice have also shown that overexpression of $M Y C$ cooperates with additional alterations in HER2 (ref. 9) and Bcl-2 (ref. 10) as well as inactivation of p53 (ref. 11) to promote tumor formation. In addition, functional inactivation of $M Y C$ in human breast cancer cells specifically inhibits distant metastasis in vivo and invasive behavior in vitro. ${ }^{12}$ Taken together, these findings implicate a role for $M Y C$ in breast cancer tumorigenesis and progression.

Amplification of $M Y C$ has been reported in breast cancer as well as in many other cancers. ${ }^{4,13,14}$ Despite numerous studies, the proportion of breast cancers reported to harbor $M Y C$ amplification ranges widely, from 1 to $94 \% .{ }^{15}$ There is also no clear consensus as to whether or not $M Y C$ amplification is always associated with overexpression of its protein product. Regardless, MYC amplification is consistently observed in more aggressive ER-negative disease and correlates with poor prognosis and distant metastasis $^{16-21}$ However, to date, a systematic analysis of $M Y C$ amplification in metastatic breast cancers has not been reported. In addition, whether the $M Y C$ amplification status may change in metastases in comparison to the corresponding primary breast tumor has also not been determined.

Our group has recently assembled unique cohorts of breast cancers from rapid autopsies and surgical specimens, in which paraffin-embedded tissues of both the initial primary and subsequent matched systemic metastasis are available for analysis. Using these unique cohorts, we have analyzed both $M Y C$ protein expression and chromosomal copy number in both primary breast tumors and matched metastases.

\section{Materials and methods}

\section{Cases and Tissue Microarray Construction}

Two cohorts of patient samples were used to create two sets of tissue microarrays for analysis. In both cohorts, paraffin-embedded tissues were available from a single patient's primary breast carcinoma and her matched hematogenous metastasis. The clinicopathological features of the initial cases of the autopsy cohort have been reported previously ${ }^{22-24}$ so they are only summarized here. For the autopsy cohort, all 15 patients studied developed terminal metastatic breast carcinoma and consented to undergo rapid autopsy for research purposes upon their death. All autopsies were performed within $4 \mathrm{~h}$ of the patient's death, and multiple metastases were harvested and fixed in formalin so as to approximate the fixation of a surgical specimen. Paraffin blocks of multiple metastases harvested at autopsy and the patient's archived primary breast carcinoma from surgical pathology were used to make single patient tissue microarrays. The arrays consisted of 99 spots, each $1.5 \mathrm{~mm}$ in diameter. For virtually all tumor samples, five spots were placed on the array to minimize sampling error. In rare lesions in which limited material was available, fewer spots were used. In total, the tissue microarrays contained 145 primary tumor spots and 778 spots derived from 180 different metastases. In addition, two separate breast cancer metastasis tissue microarrays were constructed composed of 10 matched primary breast cancers and corresponding solitary metastases sampled not at autopsy but rather in routine surgical resections. The clinicopathological features of this surgical cohort have been reported. ${ }^{25}$ The distribution of metastases of the 10 metastatic sites was as follows: 6 brain, 3 lung and 1 ovary. Similar to the autopsy cohort, for all tumor samples, at least five spots were placed on the array to minimize sampling error. This totaled 50 primary tumor spots and 86 metastatic tumor spots.

Immunohistochemical analysis of ER, PR, HER2/ neu, CK5/6 and EGFR expression was assessed, and the cases were characterized as either luminal, basal-like or HER2 based on published criteria, which correlate with groups defined by gene expression profiling. The immunohistochemical surrogate profiles are as follows:

Luminal A tumors: ER positive, HER2 negative

Luminal B tumors: ER positive, HER2 positive

HER2 tumors: ER negative, PR negative, HER2 positive

Basal-like tumors: ER negative, PR negative, HER2 negative, CK5/6 positive and/or EGFR positive.

Labeling for ER, PR and HER2 was also evaluated in metastases to determine loss or gain of expression. Cases with a luminal A phenotype that lost expression of either ER and/or $\mathrm{PR}$ in metastases were categorized as luminal $A$, loss cases. ${ }^{23-25}$

\section{Immunohistochemistry for MYC Protein}

Immunohistochemical staining for $M Y C$ was performed on all matched primary and metastatic 
autopsy and surgical specimens. Immunohistochemical staining was performed on $5-\mu \mathrm{m}$-thick sections from formalin-fixed and paraffin-embedded tissue microarrays. Slides were deparaffinized and subjected to antigen retrieval by steaming for $40 \mathrm{~min}$ in EDTA solution (Zymed, South San Francisco, CA, USA). Slides were incubated with the rabbit monoclonal anti-MYC antibody (clone Y69, Epitomics, Burlingame, CA, USA) at 1:300 dilution overnight at $4{ }^{\circ} \mathrm{C}$. An anti-rabbit IgG antibody-HRP conjugate (PowerVision + Leica Microsystems, Buffalo Grove, IL, USA) was used as secondary antibody for $30 \mathrm{~min}$ at room temperature. Staining was visualized using 3,3'-diamino-benzidine (Sigma, Saint Louis, MO, USA) and slides were counterstained with hematoxylin. Mouse prostatic tissues from transgenic mice overexpressing human $M Y C$ were used as positive controls. ${ }^{26}$ The predominant labeling was nuclear in location and only nuclear labeling was scored. Immunohistochemical staining results were assigned a score by multiplying intensity $(0-3)$ of positive staining with the number of stained cells. Scores were then subclassified into low (1-100), moderate (101-200) and high (201-300) expressors.

\section{Fluorescence In Situ Hybridization for MYC Amplification}

Dual-label fluorescence in situ hybridization was performed on each tissue microarray using standard techniques. ${ }^{13}$ Briefly, 5- $\mu$ m-thick slides were deparaffinized, dehydrated, incubated in $2 \times \mathrm{SSC}$ at $75^{\circ} \mathrm{C}$ for $15 \mathrm{~mm}$. Slides were then digested in pepsin solution ( $(4 \mathrm{mg} / \mathrm{ml}$ in $0.9 \% \mathrm{NaCl}(\mathrm{pH} 1.5))$ for $15 \mathrm{~min}$ at $37^{\circ} \mathrm{C}$ rinsed in $2 \times \mathrm{SSC}$ at room temperature for $5 \mathrm{~min}$, and air-dried. Directly labeled fluorescent DNA probes (Abbott Molecular, Abbott Park, IL, USA) for the centromere region of chromosome 8 (green) and the genomic locus of $M Y C$ (red) were used in this study. Hybridization was performed by first denaturing both probes and target DNA by incubation in an $80^{\circ} \mathrm{C}$ oven for $5 \mathrm{~min}$ and then incubating each slide at $37^{\circ} \mathrm{C}$ overnight. Slides were postwashed in $1.5 \mathrm{M}$ urea/0.1 $\times$ SSC at $45^{\circ} \mathrm{C}$ for $30 \mathrm{~min}$ and in $2 \times \mathrm{SSC}$ at room temperature for 2 min. Nuclei were counterstained with 4,6-diamidino-2-phenylindole and anti-fade compound $p$-phenylenediamine.

Of the autopsy cohort, all primary tumors and metastases were successfully stained. In the surgical specimens, staining was successful in 9 of 10 primaries and 10 of 10 metastases. Nuclei of up to 50 tumor cells were scored for each signal from each tumor core. The ratio of $M Y C$ to centromere 8 was calculated for each core, and ratios for each tumor and metastasis were averaged. As per previously published criteria, amplification of $M Y C$ was defined as an $M Y C$ to centromere 8 ratio of $>2.2$. A ratio of 1.3-2.2 was considered an $M Y C$ duplication and $<1.3$ was normal. ${ }^{13}$

\section{Results}

\section{Cases}

The cohorts consisted of 15 autopsy and 10 surgical cases. The clinicopathological features for both patient groups are summarized in Table 1. In the autopsy cohort (cases 1-15), patient age at diagnosis ranged from 28 to 71 years (mean 46 years) and age of death ranged from 36 to 79 years (mean 52 years). All of the primaries except 1 were invasive ductal carcinoma and the other was an invasive lobular carcinoma. On the basis of hormone receptor status, HER2/neu, CK5/6 and EGFR protein expression, the primaries could be further subcategorized into luminal A $(n=8)$, basal-type $(n=5)$, and HER2 positive $(n=2)$. In the corresponding metastases, 4 of $8(50 \%)$ luminal A cases demonstrated ER and/or PR loss, and thus were classified as luminal loss cases. In addition, metastases from 1 of 2 HER2positive cases showed subsequent HER2 loss. A mean of four sites of metastasis was sampled at autopsy and analyzed on tissue microarray (range 1-8 sites). The sites of metastases varied widely among the autopsy cases; however, the most common organs involved included the liver $(n=10$, $67 \%)$, lung $(n=9,60 \%)$ and adrenal gland $(n=6$, $40 \%$ ). Axillary lymph node metastases were also present in 6 of 15 cases at the time of diagnosis and 4 of 15 cases postmortem.

In the surgical cohort (cases 16-25), the patients ranged in age at diagnosis from 33 to 53 years (mean 42 years) and from 33 to 59 years (mean 46 years) when the corresponding metastasis was collected. Six of the $10(60 \%)$ patients died after specimens were collected with the age of death ranging from 35 to 59 years (mean 47 years). All cases were invasive ductal carcinomas with the exception of case 19, which was an invasive lobular carcinoma. Primaries consisted of four luminal A, four basal-type and two HER2/neu-positive cases. The molecular subtype of the corresponding surgical metastases remained unchanged. Only a single metastasis for each primary was available for review. Sites of metastatic involvement consisted of six brain, three lung and one ovary.

\section{Immunohistochemical Staining}

The results of $M Y C$ immunohistochemistry are summarized in Table 2 . In the autopsy cohort, 12 of $15(80 \%)$ primaries demonstrated $M Y C$ labeling, but the majority of the primaries were low expressors (8 of 12, 67\%). In addition, four of the low expressing cases showed only focal and weak labeling. Analysis of postmortem metastases from 11 of $12(92 \%)$ MYC-positive primaries surprisingly exhibited absent $(n=7)$ or diminished $(n=4)$ labeling. Further, the metastases that showed detectable MYC labeling were focal and weak. With the exception of a pleural metastasis, case 13 was the 
Table 1 Clinicopathological information and corresponding fluorescence in situ hybridization analysis of both autopsy and surgical cohorts

\begin{tabular}{|c|c|c|c|c|c|c|c|}
\hline Case & $\begin{array}{c}\text { Age at diagnosis } \\
\text { (years) }\end{array}$ & $\begin{array}{l}\text { Primary tumor } \\
\text { type and grade }\end{array}$ & $\begin{array}{l}\text { Stage at } \\
\text { diagnosis }\end{array}$ & $\begin{array}{l}\text { Age at death } \\
\text { (years) }\end{array}$ & $\begin{array}{l}\text { Molecular } \\
\text { subtype }\end{array}$ & $\begin{array}{l}P B C \\
C-m y c\end{array}$ & $\begin{array}{l}M B C \\
c-m y c\end{array}$ \\
\hline 1 & 54 & ILC, grade 2 & T2N0M0 & 65 & LA loss & NDA & NDA \\
\hline 2 & 59 & IDC, grade 2 & T1N0M0 & 68 & LA & NDA & NDA \\
\hline 3 & 48 & IDC, grade 3 & T2N3M0 & 54 & LA & NDA & NDA \\
\hline 4 & 71 & IDC, grade 3 & T2NoMX & 79 & BLC & NDA & NDA \\
\hline 5 & 35 & IDC, grade 3 & T2N1MX & 36 & HER2 loss & NDA & NDA \\
\hline 6 & 57 & IDC, grade 2 & T4N1M1 & 58 & LA loss & NDA & NDA \\
\hline 7 & 33 & IDC, grade 3 & T3N1M0 & 37 & BLC & DUP & DUP \\
\hline 8 & 38 & IDC, grade 3 & T2NXMX & 43 & LA & DUP & DUP \\
\hline 9 & 42 & IDC, grade 3 & T3N1M1 & 47 & HER2 & NDA & AMP \\
\hline 10 & 51 & IDC, grade 3 & T2NoMo & 53 & BLC & DUP & AMP \\
\hline 11 & 28 & IDC, grade 3 & T2N1MX & 38 & LA & DUP & AMP \\
\hline 12 & 33 & IDC, grade 3 & T1N1MX & 37 & BLC & DUP & AMP \\
\hline 13 & 56 & IDC, grade 2 & T2N1M0 & 61 & LA loss & AMP & AMP \\
\hline 14 & 40 & IDC, grade 3 & T1N1M0 & 48 & LA loss & AMP & AMP \\
\hline 15 & 47 & IDC, grade 3 & T2N1MX & 48 & BLC & AMP & AMP \\
\hline 16 & 33 & IDC, grade 3 & T3N1M1 & Still alive & HER2 & NDA & NDA \\
\hline 17 & 50 & IDC, grade 3 & T2N1MX & Still alive & LA & NDA & NDA \\
\hline 18 & 34 & IDC, grade 3 & T2N2M1 & 35 & BLC & DUP & AMP \\
\hline 19 & 38 & ILC, grade not reported & T1cNoMX & 44 & LA & NDA & NDA \\
\hline 20 & 45 & IDC, grade not reported & Unknown & Still alive & LA & NDA & AMP \\
\hline 21 & 36 & IDC, grade 2 & T1cNoMX & Still alive & HER2 & Failed & AMP \\
\hline 22 & 39 & IDC, grade 3 & T2NOMX & 45 & BLC & NDA & DUP \\
\hline 23 & 53 & IDC, grade not reported & Unknown & 55 & BLC & AMP & AMP \\
\hline 24 & 38 & IDC, grade 3 & T1cN0MX & 43 & BLC & NDA & NDA \\
\hline 25 & 53 & IDC, grade 3 & T2N2MX & 59 & LA & NDA & NDA \\
\hline
\end{tabular}

Abbreviations: BLC, basal-like carcinoma; HER2, HER2 amplified; HER2 loss, HER2 loss in metastases; IDC, invasive ductal carcinoma; ILC, invasive lobular carcinoma; LA, luminal A; LA Loss, luminal A, ER/PR loss in metastases; MBC, metastatic breast cancer; NDA, no duplication or amplification; PBC, primary breast cancer.

Table 2 c-Myc immunohistochemical staining for individual primary breast cancers and corresponding metastases

Case Primary Ax. lymph Ax. lymph Adrenal Bladder Brain Colon Diaph. Gall. Heart Liver Lung Oment. Ovary Pleura Med. Skin Spleen node node

\begin{tabular}{|c|c|c|c|c|c|c|c|c|c|c|c|c|c|c|c|c|c|c|}
\hline 1 & 4 & - & - & - & - & - & - & - & - & 0 & 0 & - & - & - & - & - & - & - \\
\hline 2 & 5 & - & - & 0 & - & 0 & - & - & - & - & 0 & 0 & - & - & 0 & - & - & - \\
\hline 3 & 0 & 0 & - & - & - & - & - & - & - & - & 0 & - & - & - & 0 & - & - & - \\
\hline 4 & 4 & - & - & 0 & - & - & - & - & - & - & 0 & 0 & 0 & - & - & 0 & - & - \\
\hline 5 & 119 & - & - & - & - & - & - & 0 & - & - & - & 0 & & 0 & 0 & 0 & - & - \\
\hline 6 & 0 & - & - & - & - & - & - & 0 & - & - & - & 0 & & - & - & - & - & - \\
\hline 7 & 225 & 240 & 0 & 0 & - & - & - & 0 & - & - & 11 & 31 & - & - & - & - & - & - \\
\hline 8 & 84 & - & - & 0 & - & - & - & - & - & - & - & 6 & - & - & - & - & - & - \\
\hline 9 & 163 & - & - & - & - & - & - & - & - & - & - & 0 & - & - & - & 0 & - & 9 \\
\hline 10 & 120 & - & 7 & 0 & - & - & - & 0 & - & - & 0 & - & 1 & - & - & 0 & - & - \\
\hline 11 & 0 & 3 & 12 & 10 & 4 & - & - & 140 & - & - & 2 & 17 & - & - & - & 28 & 15 & - \\
\hline 12 & 9 & 0 & - & - & - & 0 & - & - & - & - & - & - & - & - & - & - & - & - \\
\hline 13 & 24 & 0 & - & - & - & - & 20 & - & - & 20 & 0 & 26 & - & - & 2 & - & - & - \\
\hline 14 & 80 & 40 & - & - & - & 0 & - & - & - & - & 8 & 3 & - & 4 & & - & - & - \\
\hline 15 & 91 & - & 0 & - & - & - & - & - & - & - & 0 & - & - & - & - & 0 & - & - \\
\hline 16 & 60 & - & - & - & - & - & - & - & - & - & - & 80 & - & - & - & - & - & - \\
\hline 17 & 0 & - & - & - & - & - & - & - & - & - & - & 65 & - & - & - & - & - & - \\
\hline 18 & 104 & - & - & - & - & 210 & - & - & - & - & - & - & - & - & - & - & - & - \\
\hline 19 & 8 & - & - & - & - & - & - & - & - & - & - & - & - & 99 & - & - & - & - \\
\hline 20 & 10 & - & - & - & - & - & - & - & - & - & - & 156 & - & - & - & - & - & - \\
\hline 21 & 0 & - & - & - & - & 139 & - & - & - & - & - & - & - & - & - & - & - & - \\
\hline 22 & 60 & - & - & - & - & 88 & - & - & - & - & - & - & - & - & - & - & - & - \\
\hline 23 & 23 & - & - & - & - & 132 & - & - & - & - & - & - & - & - & - & - & - & - \\
\hline 24 & 12 & - & - & - & - & 78 & - & - & - & - & - & - & - & - & - & - & - & - \\
\hline 25 & 163 & - & - & - & - & 84 & - & - & - & - & - & - & - & - & - & - & - & - \\
\hline
\end{tabular}

Abbreviations: Ax., axillary; diaph., diaphragm; gall., gallbladder; med., mediastinum; oment., omentum.

only case with comparable labeling to the primary. Of the three negative primaries, metastases harvested at autopsy for case 11 showed increased labeling as compared with the primary and axillary lymph node metastases harvested at the time of diagnosis, respectively. In fact, case 11 was the only case out of 15 overall $(7 \%)$ that demonstrated an increase in $M Y C$ labeling. Labeling among the 
metastases for case 11 varied widely and ranged from 2 to 140 with the highest labeling in the diaphragm.

MYC labeling was identified in 8 of $10(80 \%)$ primaries in the surgical resection specimen cohort. Similar to the autopsy cohort, the majority of cases scored within the low expressor range (6 of $8,75 \%)$, with the other $2(25 \%)$ being moderate expressors. Two of the low scoring cases showed focal and weak labeling. However, in contrast to the autopsy cases, all of the corresponding metastases (10 of 10) were $M Y C$ positive, including Cases 17 and 21, where the primary was $M Y C$ negative. Immunohistochemical scores of the metastases ranged from 78 to 210 and were subclassified as follows: six low expressor, three moderate expressor and one high expressor. Overall, the scores increased in the metastases as compared with the primaries in 9 of $10(90 \%)$ cases. Only case 25 demonstrated a twofold reduction in immunohistochemical score.

\section{Fluorescence In Situ Hybridization Analysis of MYC Amplification}

Assessment of $M Y C$ gene amplification on both autopsy and surgical cohorts is summarized in Tables 1 and 3. All 15 autopsy primaries were suitable for fluorescence in situ hybridization analysis and representative results are shown in Figure 1 and 2. Seven of $15(45 \%)$ primaries demonstrated no evidence of a selective gain of $M Y C$ gene copy number, although of these, 1 case had a gain of centromere 8 and an equivalent gain of $M Y C$. The eight remaining primaries (55\%) demonstrated an additional increase of $M Y C$ relative to centromere 8 with a 1.59- to 4.05-fold higher copy number. Among the eight tumors with an additional increase, five (33\% of the total 15) demonstrated duplication of $M Y C$ and three (20\% of the total 15$)$ demonstrated amplification of $M Y C$. An additional increase in $M Y C$ was found in nine metastases harvested at autopsy and ranged from 1.51- to 6.13fold higher than centromere 8 . The metastases in Cases 7 and 8 remained unchanged from the corresponding primaries and demonstrated $M Y C$ duplication. Cases 13, 14 and 15 also remained unchanged and showed amplification of MYC. However, Cases 9, 10, 11 and 12 acquired $M Y C$ amplification. The primary in case 9 showed a normal $M Y C$ copy number, while a duplication was present in the primaries for Cases 10, 11 and 12 . Importantly, remarkably little heterogeneity was identified between different metastases harvested postmortem from the same patient. Except for case 11, variations in $M Y C$ among metastases remained within 1 gene copy number.

Of the 10 cases within the surgical cohort, fluorescence in situ hybridization was performed successfully on 9 primaries and all 10 metastases. Seven of nine evaluable primary tumors demonstrated no evidence of $M Y C$ amplification or duplication, although four showed equally increased $M Y C$ and chromosome 8 copy number. An additional increase in $M Y C$ was observed in two of nine primary tumors: Cases 18 (duplication) and 23 (amplification). Four of nine (45\%) corresponding

Table 3 Fluorescence in situ MYC to centromere 8 ratios for individual primary breast cancers and corresponding metastases

Case Primary Ax. lymph Ax. lymph Adrenal Bladder Brain Colon Diaph. Gall. Heart Liver Lung Oment. Ovary Pleura Med. Skin Spleen node node

\begin{tabular}{|c|c|c|c|c|c|c|c|c|c|c|c|c|c|c|c|c|c|}
\hline 1.07 & - & - & - & - & - & - & - & - & 1.03 & 1.06 & - & - & - & - & - & - & - \\
\hline 1.02 & - & - & 1.05 & - & 1.06 & - & - & - & - & 1.05 & 1.15 & - & - & 1.02 & - & - & - \\
\hline 1.06 & 1.05 & - & - & - & - & - & - & - & - & 1.05 & - & - & - & 1.11 & - & - & - \\
\hline 1.13 & - & - & 1.02 & - & - & - & - & - & - & 1.04 & 1.03 & 1.05 & - & - & 1.04 & - & - \\
\hline 1.09 & - & - & - & - & - & - & 1.01 & - & - & - & 1.00 & & 1.02 & 1.02 & 1.00 & - & - \\
\hline 1.00 & - & - & - & - & - & - & 0.99 & - & - & - & 1.00 & & - & - & - & - & - \\
\hline 1.68 & 1.72 & 1.57 & 1.51 & - & - & - & 1.51 & - & - & 1.61 & 1.68 & - & - & - & - & - & - \\
\hline 1.59 & - & - & 1.56 & - & - & - & - & - & - & - & 1.60 & - & - & - & - & - & - \\
\hline 1.04 & - & - & - & - & - & - & - & - & - & - & 5.78 & - & - & - & 5.52 & - & 5.19 \\
\hline 2.17 & - & 5.77 & 5.41 & - & - & - & 6.13 & - & - & 5.19 & - & 5.58 & - & - & 5.67 & - & - \\
\hline 2.03 & 2.09 & 5.12 & 6.05 & 4.28 & - & - & 3.78 & - & - & 4.39 & 5.85 & - & - & - & 4.17 & 5.01 & - \\
\hline 2.11 & 2.22 & - & - & - & 5.19 & - & - & - & - & - & - & - & - & - & - & - & - \\
\hline 2.59 & 2.69 & - & - & - & - & 3.65 & - & - & 3.48 & 3.10 & 3.70 & - & - & 3.40 & - & - & - \\
\hline 2.96 & 3.11 & - & - & - & 3.91 & - & - & - & - & 3.34 & 3.31 & - & 3.50 & & - & - & - \\
\hline 4.05 & - & 4.02 & - & - & - & - & - & - & - & 4.15 & - & - & - & - & 4.10 & - & - \\
\hline 1.04 & - & - & - & - & - & - & - & - & - & - & 1.04 & - & - & - & - & - & - \\
\hline 0.99 & - & - & - & - & - & - & - & - & - & - & 1.03 & - & - & - & - & - & - \\
\hline 1.50 & - & - & - & - & 5.09 & - & - & - & - & - & - & - & - & - & - & - & - \\
\hline 1.02 & - & - & - & - & - & - & - & - & - & - & - & - & 1.00 & - & - & - & - \\
\hline 1.01 & - & - & - & - & - & - & - & - & - & - & 5.07 & - & - & - & - & - & - \\
\hline Failed & - & - & - & - & 6.50 & - & - & - & - & - & - & - & - & - & - & - & - \\
\hline 1.24 & - & - & - & - & 1.61 & - & - & - & - & - & - & - & - & - & - & - & - \\
\hline 2.35 & - & - & - & - & 2.89 & - & - & - & - & - & - & - & - & - & - & - & - \\
\hline 1.09 & - & - & - & - & 1.11 & - & - & - & - & - & - & - & - & - & - & - & - \\
\hline 1.13 & - & - & - & - & 1.15 & - & - & - & - & - & - & - & - & - & - & - & - \\
\hline
\end{tabular}

Abbreviations: Ax., axillary; diaph., diaphragm; gall., gallbladder; med., mediastinum, oment., omentum. 

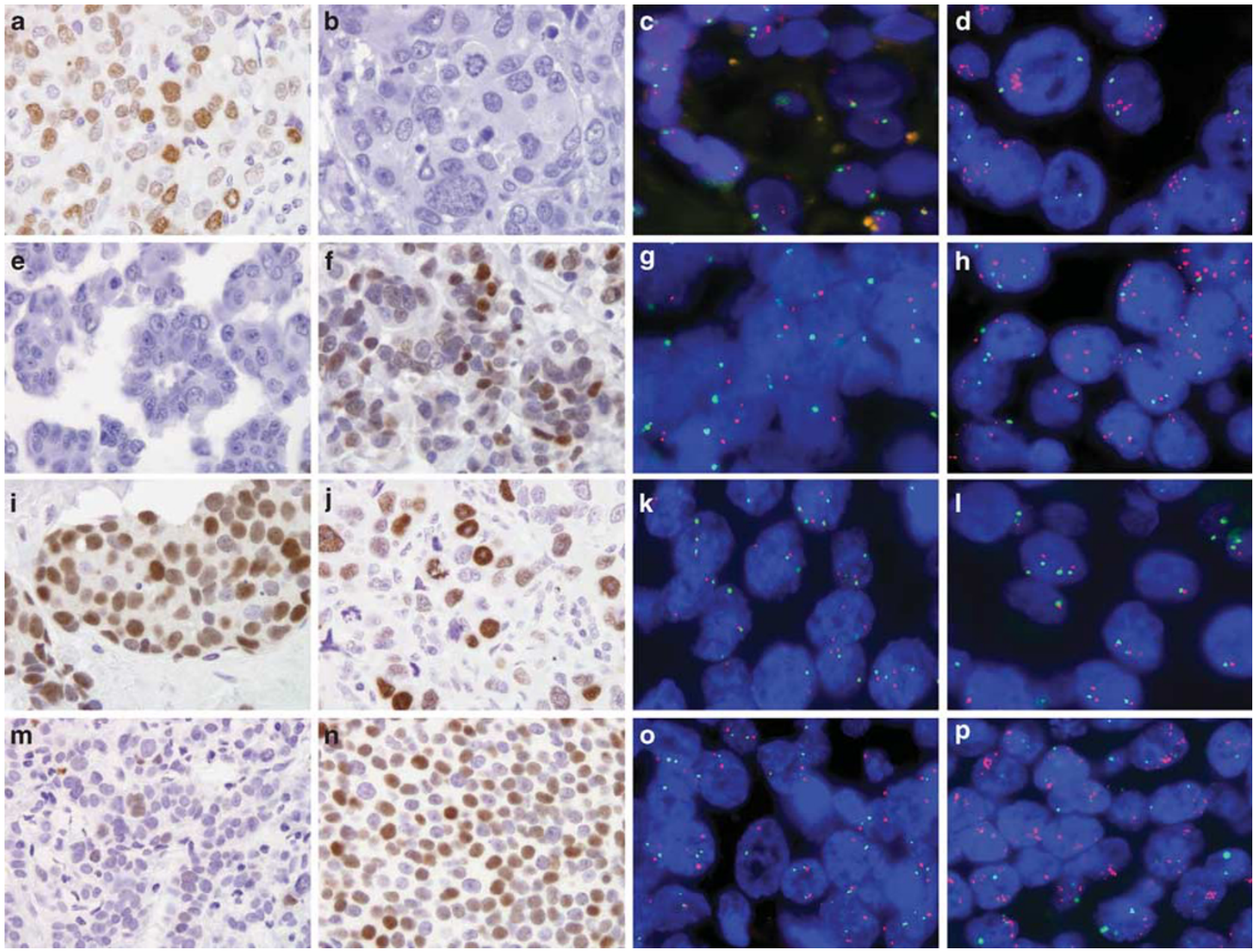

Figure 1 Representative MYC immunohistochemical staining and corresponding two-color fluorescence in situ hybridization (MYC in red and centromere 8 in green) in matched primary and metastatic breast cancers from rapid autopsies (a-h) and surgical specimens (i-p). The primary in case 9 was $M Y C$ positive by immunolabeling and scored as a moderate expressor (a), while the corresponding metastasis was $M Y C$ negative (b). This result was inconsistent with fluorescence in situ hybridization, where the metastasis acquired $M Y C$ amplification (d), but no abnormalities in $M Y C$ copy number were detected in the primary (c). In contrast, the primary in case 11 was $M Y C$ negative (e), while the metastasis was $M Y C$ positive (f, moderate expressor). By fluorescence in situ hybridization, $M Y C$ was duplicated in the primary (g) and amplified in the metastasis (h). In case 25, both the primary (i) and metastasis (j) were $M Y C$ positive. However, only half the number of cells stained for $M Y C$ in the metastasis as compared with the primary. By fluorescence in situ hybridization, $M Y C$ copy number relative to centromere 8 remained unchanged (k, primary and $\mathbf{l}$, metastasis). For case 20, an increase in $M Y C$ immunostaining was observed in the metastasis (n, moderate expressor) as compared with the primary (m, low expressor). Further, while MYC was neither duplicated nor amplified in the primary (o), $M Y C$ was amplified in the metastasis (p). See Tables 2 and 3 for c-myc immunohistochemistry and fluorescence in situ hybridization MYC to centromere 8 ratios for each primary and corresponding metastases.

metastases demonstrated an additional increase in $M Y C$. The metastasis in case 23 remained $M Y C$ amplified; however, comparing the metastases to the primary, Cases 18 and 20 acquired amplification. Further, the metastasis of case 22 showed a duplication of $M Y C$, while no alterations were identified in the corresponding primary.

\section{Axillary Lymph Node Metastases Harvested at Initial Surgery and at Autopsy are Different}

Among the tumors that acquired amplification, an interesting trend was observed when comparing the axillary lymph node metastasis harvested with the primary tumor at the time of initial surgery with the axillary lymph nodes harvested at autopsy. In case 10, axillary lymph nodes were harvested at autopsy only, and these displayed the acquired $M Y C$ amplification of the systemic metastases. In case 11, the axillary lymph nodes harvested with the primary at initial surgery demonstrated the $M Y C$ duplication seen in the primary, while those harvested at autopsy demonstrated the $M Y C$ amplification seen in the systemic metastases. In case 12 , the axillary metastasis harvested with the primary at initial surgery showed the duplication seen in the primary, not the amplification seen in the systemic metastases harvested at autopsy. Finally, in case 13, which showed amplification in the primary and a higher level of amplification in the systemic metastasis, the axillary lymph node harvested with the primary 

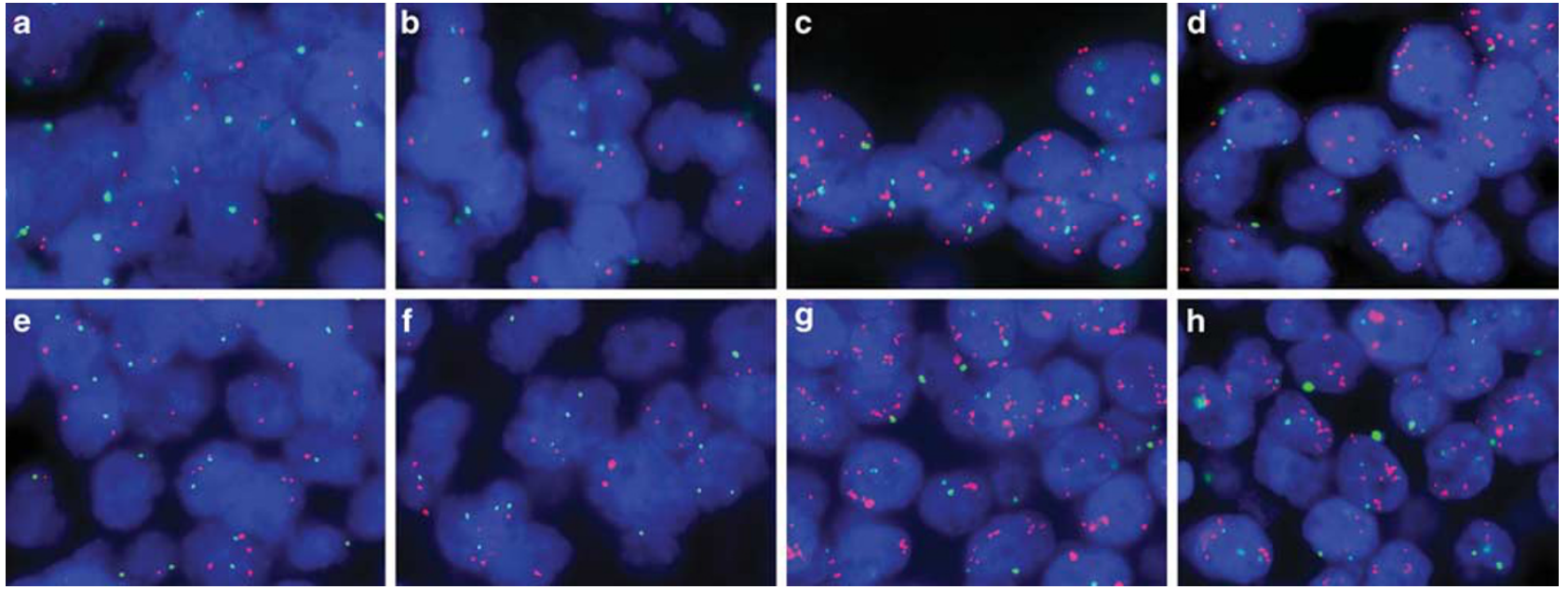

Figure 2 Comparison of $M Y C$ copy number in primary breast cancer, lymph node metastases upon initial diagnosis, and subsequent metastases on autopsy by two-color fluorescence in situ hybridization (MYC in red and centromere 8 in green) for cases 11 (a-d) and 12 $(\mathbf{e}-\mathbf{h})$. At the time of diagnosis, both the primary (a, case 11; e, case 12) and axillary lymph node metastases (b, case 11; f, case 12) were characterized by a similar MYC duplication. However, systemic metastases harvested at autopsy for both cases (c and $\mathbf{d}$, case 11; $\mathbf{g}$ and $\mathbf{h}$, case 12) acquired an MYC amplification. Metastases for case 11 include axillary lymph node (c) and lung (d), while case 12 include two separate brain metastases ( $\mathbf{g}$ and $\mathbf{h}$ ). See Table 3 for fluorescence in situ MYC to centromere 8 ratios for each primary and corresponding metastases.

showed a similar level of $M Y C$ amplification as did the primary. Hence, while the numbers are small, axillary lymph nodes harvested at initial surgery reflected the $M Y C$ status of the primary tumor, while those harvested at the time of autopsy reflected the status of the systemic metastases.

\section{Correlation of MYC Amplification with Protein Overexpression}

Given the minimal $M Y C$ immunohistochemical labeling in the autopsy metastases, which we believe represents a fixation delay artifact (see Discussion), ${ }^{27}$ we excluded these samples from our correlation analysis. The mean MYC immunohistochemical score of those tumors showing no evidence of $M Y C$ amplification was 42 , while that of tumors showing duplication was 88 and those showing amplification was 65. Of the three surgical cases, which acquired amplification or duplication, the $M Y C$ immunohistochemical score increased from the primary to the metastasis. However, the primary tumor with the highest $M Y C$ immunohistochemical score (case 25) showed neither amplification nor duplication. Hence, while there was a trend for $M Y C$ amplification and immunohistochemical labeling to correlate, the correlation was clearly imperfect.

\section{Discussion}

The extent of $M Y C$ involvement in breast cancer tumorigenesis has been highly debated. Several studies have examined $M Y C$ gene amplification and protein overexpression in breast cancers; however, the data have been conflicting. For instance, frequencies among different reports vary from 50 to $100 \%$ and 1 to $94 \%$ for protein overexpression and gene amplification, respectively. ${ }^{15}$ The source of such variation may lie at least in part due to the inconsistencies of the assay reagents and methods. For example, most studies assessing gene amplification have been performed using Southern blotting or PCR techniques. Both may suffer from normal cell contamination of the tumor sample, resulting in large fluctuations in copy number. ${ }^{28}$ Similar issues have plagued immunohistochemical evaluation of $M Y C$. Several $M Y C$ antibodies to various epitopes and modifications have been generated and utilized; however, most of these have not been rigorously evaluated using appropriate controls. Our study was designed to overcome both of these problems. First, MYC amplification was addressed in situ by fluorescence in situ hybridization, removing the variable of normal cell contamination. Second, we used a recently developed rabbit monoclonal antibody to $M Y C$ for immunohistochemistry. In conjunction with a highly sensitive immunohistochemical protocol and genetically defined control experiments, this allows a validated assessment of $M Y C$ protein expression as previously described. ${ }^{26}$ Although the correlation between $M Y C$ amplification and various clinicopathological parameters has been inconsistent, there is a general agreement that $M Y C$ amplification in primary breast carcinomas is a poor prognostic factor, which correlates with the development of distant metastases and decreased overall survival. ${ }^{16-21}$ However, to date, whether $M Y C$ amplification or overexpression develops in breast cancer metastases has not been addressed. Further, assessment of MYC status in both primaries and corresponding metastases has also not been 
performed. Therefore, we analyzed both $M Y C$ protein overexpression via validated immunohistochemistry and chromosomal alterations via the 'gold standard,' fluorescence in situ hybridization, in two cohorts of matched primary and metastases.

Several studies have shown an association between $M Y C$ amplification and ER-negative breast cancers, especially basal-like tumors. ${ }^{29-31}$ Basal-like cancers are generally aggressive, high-grade tumors, prone to develop hematogenous metastases, associated with shorter survival and relatively high mortality rate. ${ }^{32}$ Therefore, it was not surprising in our study to find $60 \%$ of primaries with gains in $M Y C$ were ER-negative, and all of these were basallike carcinomas. In addition, $79 \%$ of metastases with increased $M Y C$ copy number were ER-negative carcinomas, which included basal-like carcinomas, HER2/neu carcinomas and luminal A carcinomas that subsequently lost ER and PR expression.

Our study shows that a significant subset of metastatic breast cancers demonstrates homogenous MYC gene amplification, which was not detected in the primary site. Importantly, the percentage of cases that acquired homogeneous $M Y C$ amplification in metastases (6 of 24 evaluable cases overall, 25\%) was greater than the percentage of primary tumors that demonstrated $M Y C$ amplification (4 of 24 evaluable cases, 16\%). The percentage of cases developing homogeneous $M Y C$ amplification in their metastases was similar in the autopsy cohort (4 of 15 , or $27 \%$ ) as in the surgical cohort ( 2 of 9 , $22 \%$ ). $M Y C$ amplification spans all of the molecular subtypes of breast cancer, in that it was found in luminal, HER2 and basal like carcinomas in this study. An important point is that $M Y C$ amplification was strikingly consistent among different metastases from the same patient. This result contrasts with our results in this same cohort for EGFR, which showed variable amplification between different metastases. ${ }^{22}$ This result also contrasts with the literature on $M Y C$ amplification in primary tumors, where heterogeneity of $M Y C$ amplification is well documented. ${ }^{33}$ These findings indicate that $M Y C$ amplification is a driver of and selected for in breast cancer metastases. We suspect that the 'unamplified' primary tumors which gave rise to metastases with homogeneous $M Y C$ amplification likely harbored small subclones with $M Y C$ amplification, and that these clones gave rise to the systemic metastases. Alternatively, amplification may have arisen in an early metastatic lesion, which then gave rise to all of the other metastases in a given patient. Unfortunately, our samples of the primary tumors in this cohort were limited, and we could not exhaustively search these primary tumors for small subclones with $M Y C$ amplification. Supporting the idea that $M Y C$ amplification provides a selective advantage for metastasis, Wolfer et $a l^{12}$ recently found that MYC coordinately regulates the express of 13 different 'poor outcome' cancer signatures, and that functional inactivation of $M Y C$ inhibits distant metastases and invasion independent of the effects upon survival, proliferation and differentiation. Hence, $M Y C$ has a plausible biological role as a driver of metastasis, and thus is a rational therapeutic target in metastatic breast cancer.

Genetic changes that distinguish metastases from primary breast carcinomas are few and far between; most expression studies have not found consistent differences between metastatic and primary tumors. Hence, homogenous $M Y C$ amplification qualifies as a bona fide genetic alteration, which correlates with breast cancer progression to metastasis. Although no prior study has evaluated $M Y C$ amplification in metastases in the manner that we have, other studies have implicated $M Y C$ amplification in neoplastic progression in primary breast tumors. For example, Robanus-Maandag et $a 3^{34}$ evaluated paired ductal carcinoma in situ and invasive ductal carcinomas for $M Y C$ amplification, and found no amplification in ductal carcinoma in situ associated with invasive ductal carcinomas that harbored $M Y C$ amplification. Aulmann et $a l^{21}$ analyzed invasive ductal carcinomas, which locally recurred, and found acquisition of $M Y C$ amplification in 6 of 38 recurrences. Similarly, Naidu et $a l^{31}$ found $M Y C$ amplification in three of nine invasive ductal carcinomas, but not the adjacent non-comedo ductal carcinoma in situ lesions. Hence, our data support prior studies of early stage disease that implicates $M Y C$ as a rare confirmed driver of neoplastic progression in breast cancer.

When evaluating our surgical cases, we found an imperfect correlation of $M Y C$ amplification with $M Y C$ immunohistochemical labeling. These results indicate that other mechanisms may affect $M Y C$ protein overexpression in breast carcinoma. Our results with the autopsy cohort are at first glance difficult to explain, given that $M Y C$ protein was decreased in the autopsy metastases, including those cases that developed $M Y C$ amplification. As the autopsy patients were refractory to therapy and $M Y C$ is a driver of cell division, a feature most therapy regimens target, an argument could be made for the selection of decreased $M Y C$ expressing tumors within this group. However, we believe that this result is more likely artifactual. We have found that the detection of $M Y C$ protein overexpression by immunohistochemistry is exquisitely fixation sensitive to a much greater degree than most proteins. For example, in a well-controlled experiment using xenografts, delayed fixation for $3 \mathrm{~h}$ resulted in a sixfold reduction in $M Y C$ staining score, while a staining for a more stable antigen-like cytokeratin was unaffected. ${ }^{27}$ Although our autopsy metastases were harvested within $4 \mathrm{~h}$ of the patient's demise, these tissues remained within the patient at body temperature until they were dissected and placed into formalin. In contrast, a surgical specimen would rest at room temperature, usually for $<1 \mathrm{~h}$, before it was placed in formalin after dissection. We suspect that the combination of increased time prior 
to fixation (eg, up to $4 \mathrm{~h} v s<1 \mathrm{~h}$ ) and increased temperature of the tissue in a rapid-autopsy specimen results in much more MYC protein degradation seen in autopsy specimens as compared with surgical resection specimens. Consequently, there was an almost complete absence of $M Y C$ immunoreactivity in the autopsy metastases. In addition, these results highlight the importance of issues related to tissue fixation, including pre-fixation time and temperature, in assessment of immunohistochemical markers. While some markers may be unaffected by fixation, others (such as MYC) are exquisitely sensitive.

Finally, while our case numbers are small, our experience with axillary lymph node metastasis detected at the time of primary surgery and at autopsy suggest that these represent two biologically different lesions. The lymph node metastases harvested at the time of initial surgery reflected the MYC status of the primary tumor. However, the lymph node metastases harvested at autopsy reflected the $M Y C$ status of the systemic metastasis. Hence, it is likely that axillary lymph node metastasis harvested at the time of primary surgery reflect the genetics of the primary tumor, while those harvested at autopsy or at the time of disseminated metastasis reflect systemic disease. There are two mechanisms that come to mind that could explain this. The first is that a small portion of amplified cancer cells are present as a subclone in the initial axillary metastasis but that these subclones would not be easily sampled by our method for tissue microarray construction. Then, after systemic treatment, these cells have a major selective growth advantage and are found as the predominant clone at autopsy in the axillary lymph nodes. Another mechanism to explain this change would be the recently proposed hypothesis of tumor reseeding. The classical model of tumor metastasis is as the primary tumor grows it gradually acquires the capacity to invade, intravasate, circulate, extravasate and colonize. This was once thought to be a unidirectional process with dissemination of tumor cells to distant sites. Recently, Kim et a ${ }^{35}$ described how circulating breast tumor cells can preferentially reinfiltrate and reseed the tumor of origin, replacing their indolent counterparts with more aggressive cells. This 'self-seeding' concept is not ill-founded. As Paget ${ }^{36}$ proposed his 'seed-and-soil' theory for metastatic spread, the notion that tumors grow in favored environmental conditions has attained broad acceptance. The idea that one such location would include the site of tumor origin is certainly logical. In addition, it is tempting to speculate that self-seeding might occur not only at the primary tumor site, but also at metastatic sites. Each site could serve as a nesting ground to generate tumor variants that repopulate other metastatic sites, thus accelerating tumor progression. In fact, a similar phenomenon has been documented to occur in patients where one malignant tumor metastasizes to a different second primary tumor, a process known as 'cross-seeding.' These occurrences are extremely rare but have been reported in breast cancers. ${ }^{37,38}$ Further, Kim et $a 1^{35}$ have shown that as in these rare cases, malignant cells will metastasize to a second tumor. Considering the connection between $M Y C$ and more aggressive phenotypes, it seems fair to postulate that cancer cells with gains in $M Y C$ could overpopulate all of patient's metastasis by self-seeding. Thus, the lack of heterogeneity in copy number among metastases and between axillary metastases and the corresponding primary at the time of diagnosis is actually not surprising.

In conclusion, this study demonstrates that gains in $M Y C$ copy number are a frequent event in breast cancer and occur relatively late in tumorigenesis. These chromosomal alterations are present more often in metastatic disease than the corresponding primary, suggesting that $M Y C$ amplification provides a selective advantage for the metastatic process. Comparison of $M Y C$ status among multiple metastases from the same patient showed little variability or organ preference. Finally, axillary lymph node metastases harvested at autopsy reflected systemic disease, whereas those identified at the time of diagnosis reflected the features of the primary tumor, supporting the hypothesis that $M Y C$ amplified tumor cells are more resistant to systemic therapy, and/or of tumor self-reseeding. These observations underscore the importance of $M Y C$ in breast cancer progression/metastasis, as well as its relevance as a potential therapeutic target in otherwise incurable metastatic disease.

\section{Acknowledgement}

The NIH SPORE in Breast Cancer at Johns Hopkins (CA 88843) supported this study.

\section{Disclosure/conflict of interest}

All authors declare no conflict of interest.

\section{References}

1 Tirkkonen M, Tanner M, Karhu R, et al. Molecular cytogenetics of primary breast cancer by CGH. Genes Chromosomes Cancer 1998;21:177-184.

2 Courjal F, Theillet C. Comparative genomic hybridization analysis of breast tumors with predetermined profiles of DNA amplification. Cancer Res 1997;57: 4368-4377.

3 Nishizaki T, DeVries S, Chew K, et al. Genetic alterations in primary breast cancers and their metastases: direct comparison using modified comparative genomic hybridization. Genes Chromosomes Cancer 1997;19:267-272.

4 Escot C, Theillet C, Lidereau R, et al. Genetic alteration of the c-myc protooncogene (MYC) in human primary breast carcinomas. Proc Natl Acad Sci U S A 1986;83: 4834-4838. 
5 Chen Y, Olopade OI. MYC in breast tumor progression. Expert Rev Anticancer Ther 2008;8:1689-1698.

6 Evan GI, Littlewood TD. The role of c-myc in cell growth. Curr Opin Genet Dev 1993;3:44-49.

7 Venditti M, Iwasiow B, Orr FW, et al. C-myc gene expression alone is sufficient to confer resistance to antiestrogen in human breast cancer cells. Int J Cancer 2002;99:35-42.

8 Wang Q, Zhang H, Kajino K, et al. BRCA1 binds c-Myc and inhibits its transcriptional and transforming activity in cells. Oncogene 1998;17:1939-1948.

9 Cardiff RD, Sinn E, Muller W, et al. Transgenic oncogene mice. Tumor phenotype predicts genotype. Am J Pathol 1991;139:495-501.

10 Jager R, Herzer U, Schenkel J, et al. Overexpression of Bcl-2 inhibits alveolar cell apoptosis during involution and accelerates c-myc-induced tumorigenesis of the mammary gland in transgenic mice. Oncogene 1997;15:1787-1795.

11 McCormack SJ, Weaver Z, Deming S, et al. Myc/p53 interactions in transgenic mouse mammary development, tumorigenesis and chromosomal instability. Oncogene 1998;16:2755-2766.

12 Wolfer A, Wittner BS, Irimia D, et al. MYC regulation of a 'poor-prognosis' metastatic cancer cell state. Proc Natl Acad Sci USA 2010;107:3698-3703.

13 Jenkins RB, Qian J, Lieber MM, et al. Detection of c-myc oncogene amplification and chromosomal anomalies in metastatic prostatic carcinoma by fluorescence in situ hybridization. Cancer Res 1997;57:524-531.

14 Visscher DW, Wallis T, Awussah S, et al. Evaluation of MYC and chromosome 8 copy number in breast carcinoma by interphase cytogenetics. Genes Chromosomes Cancer 1997;18:1-7.

15 Deming SL, Nass SJ, Dickson RB, et al. C-myc amplification in breast cancer: a meta-analysis of its occurrence and prognostic relevance. Br J Cancer 2000;83:1688-1695.

16 Berns EM, Klijn JG, van Putten WL, et al. c-myc amplification is a better prognostic factor than HER2/ neu amplification in primary breast cancer. Cancer Res 1992;52:1107-1113.

17 Berns EM, Foekens JA, van Putten WL, et al. Prognostic factors in human primary breast cancer: comparison of c-myc and HER2/neu amplification. J Steroid Biochem Mol Biol 1992;43:13-19.

18 Borg A, Baldetorp B, Ferno M, et al. c-myc amplification is an independent prognostic factor in postmenopausal breast cancer. Int J Cancer 1992;51:687-691.

19 Lonn U, Lonn S, Nilsson B, et al. Prognostic value of erb-B2 and myc amplification in breast cancer imprints. Cancer 1995;75:2681-2687.

20 Rummukainen JK, Salminen T, Lundin J, et al. Amplification of c-myc oncogene by chromogenic and fluorescence in situ hybridization in archival breast cancer tissue array samples. Lab Invest 2001;81: 1545-1551.

21 Aulmann S, Adler N, Rom J, et al. c-myc amplifications in primary breast carcinomas and their local recurrences. J Clin Pathol 2006;59:424-428.

22 Wu JM, Fackler MJ, Halushka MK, et al. Heterogeneity of breast cancer metastases: comparison of therapeutic target expression and promoter methylation between primary tumors and their multifocal metastases. Clin Cancer Res 2008;14:1938-1946.

23 Cimino A, Halushka M, Illei P, et al. Epithelial cell adhesion molecule (EpCAM) is overexpressed in breast cancer metastases. Breast Cancer Res Treat 2010;123:701-708.

24 Subhawong AP, Nassar $\mathrm{H}$, Halushka MK, et al. Heterogeneity of Bcl-2 expression in metastatic breast carcinoma. Mod Pathol 2010;23:1089-1096.

25 Cimino-Mathews A, Hicks J, Illei P, et al. Androgen receptor expression is usually maintained in initial surgically-resected breast cancer metastases, but often lost in terminal metastases found at autopsy. Human Pathol 2011 (in press).

26 Gurel B, Iwata $\mathrm{T}$, Koh CM, et al. Nuclear MYC protein overexpression is an early alteration in human prostate carcinogenesis. Mod Pathol 2008;21: 1156-1167.

27 Gurel B, Iwata T, Dalrymple S, et al. Fixation delay adversely impacts androgen receptor staining. Biospecimen Research Network Symposium edn, Vol. Washington, DC, 2009.

28 Nesbit CE, Tersak JM, Prochownik EV. MYC oncogenes and human neoplastic disease. Oncogene 1999;18: 3004-3016.

29 Sorlie T, Perou CM, Tibshirani R, et al. Gene expression patterns of breast carcinomas distinguish tumor subclasses with clinical implications. Proc Natl Acad Sci USA 2001;98:10869-10874.

30 Alles MC, Gardiner-Garden M, Nott DJ, et al. Metaanalysis and gene set enrichment relative to er status reveal elevated activity of MYC and E2F in the 'basal' breast cancer subgroup. PLoS One 2009;4:e4710.

31 Naidu R, Wahab NA, Yadav M, et al. Protein expression and molecular analysis of c-myc gene in primary breast carcinomas using immunohistochemistry and differential polymerase chain reaction. Int J Mol Med 2002;9:189-196.

32 Rakha EA, Reis-Filho JS, Ellis IO. Basal-like breast cancer: a critical review. J Clin Oncol 2008;26: 2568-2581.

33 Glockner S, Buurman H, Kleeberger W, et al. Marked intratumoral heterogeneity of c-myc and cyclinD1 but not of c-erbB2 amplification in breast cancer. Lab Invest 2002;82:1419-1426.

34 Robanus-Maandag EC, Bosch CA, Kristel PM, et al. Association of C-MYC amplification with progression from the in situ to the invasive stage in C-MYCamplified breast carcinomas. J Pathol 2003;201:75-82.

35 Kim MY, Oskarsson T, Acharyya S, et al. Tumor selfseeding by circulating cancer cells. Cell 2009;139: 1315-1326.

36 Paget S. The distribution of secondary growths in cancer of the breast. Cancer Metastasis Rev 1989;8: 98-101.

37 Engel J, Eckel R, Kerr J, et al. The process of metastasisation for breast cancer. Eur J Cancer 2003;39: 1794-1806.

38 Doron Y, Gruszkiewicz J. Metastasis of invasive carcinoma of the breast to an extradural meningioma of the cranial vault. Cancer 1987;60:1081-1084. 